\title{
Hypoxia-induced miR-1260b regulates vascular smooth muscle cell proliferation by targeting GDF11
}

\author{
Minhyeong Seong ${ }^{1} \mathcal{E}$ Hara Kang ${ }^{1,2, *}$ \\ ${ }^{1}$ Division of Life Sciences, College of Life Sciences and Bioengineering, Incheon National University, Incheon $22012,{ }^{2}$ Institute for New \\ Drug Development, Incheon National University, Incheon 22012, Korea
}

\begin{abstract}
Vascular smooth muscle cells (VSMCs) are a unique cell type that has unusual plasticity controlled by environmental stimuli. As an abnormal increase of VSMC proliferation is associated with various vascular diseases, tight regulation of VSMC phenotypes is essential for maintaining vascular homeostasis. Hypoxia is one environmental stress that stimulates VSMC proliferation. Emerging evidence has indicated that microRNAs (miRNAs) are critical regulators in the hypoxic responses of VSMCs. Therefore, we previously investigated miRNAs modulated by hypoxia in VSMCs and found that miR-1260b is one of the most upregulated miRNAs under hypoxia. However, the mechanism that underlies the regulation of VSMCs via miR-1260b in response to hypoxia has not been explored. Here we demonstrated that hypoxia-induced miR-1260b promotes VSMC proliferation. We also identified growth differentiation factor 11 (GDF11), a member of the TGF- $\beta$ superfamily, as a novel target of miR-1260b. miR-1260b directly targets the 3'UTR of GDF11. Downregulation of GDF11 inhibited Smad signaling and consequently enhanced the proliferation of VSMCs. Our findings suggest that miR-1260b-mediated GDF11Smad-dependent signaling is an essential regulatory mechanism in the proliferation of VSMCs, and this axis is modulated by hypoxia to promote abnormal VSMC proliferation. Therefore, our study unveils a novel function of miR-1260b in the pathological proliferation of VSMCs under hypoxia. [BMB Reports 2020; 53(4): 206-211]
\end{abstract}

\section{INTRODUCTION}

Vascular smooth muscle cells (VSMCs) have a unique ability to turn into a contractile or synthetic phenotype in response to microenvironmental stimuli (1). Contractile VSMCs are characterized by

*Corresponding author. Tel: +82-32-835-8238; Fax: +82-32-8350763; E-mail: harakang@inu.ac.kr

https://doi.org/10.5483/BMBRep.2020.53.4.136

Received 8 May 2019, Revised 24 June 2019, Accepted 30 August 2019

Keywords: GDF11, Hypoxia, microRNA, miR-1260b, Smad signaling, Vascular smooth muscle cell a very low rate of proliferation, migration and expression of SMC contractile-associated genes, such as smooth muscle $\alpha$-actin ( $\alpha$-SMA), SM22 $\alpha$ and calponin (2). In contrast, synthetic VSMCs demonstrate increased proliferation and migration. The transforming growth factor-beta (TGF- $\beta$ ) superfamily of growth factors and the platelet-derived growth factor (PDGF) are involved primarily in the regulation of VSMC phenotypes $(3,4)$. The TGF- $\beta$ signaling pathway has been demonstrated to promote the contractile phenotype by inhibiting proliferation and migration of VSMCs (4). In contrast, the PDGF signaling pathway leads the synthetic phenotype by stimulating VSMC proliferation and migration (3). As dysregulation of VSMC phenotypes is associated with vascular diseases, VSMC proliferation and differentiation are dynamically regulated under physiological or pathological conditions.

Hypoxia is a unique environmental stress that affects cellular functions, such as proliferation, apoptosis and DNA repair (5). In VSMCs, hypoxia stimulates proliferation and can lead to vascular diseases including pulmonary artery hypertension (6). Under hypoxia, gene expression is regulated by master transcription factors, hypoxia-inducible factors (HIF) $(7,8)$. In addition, specific microRNAs (miRNAs), termed hypoxamirs, regulate gene expression through posttranscriptional mechanisms in response to hypoxia (9). Several miRNAs have emerged as crucial regulators of the VSMC phenotype by repressing their target mRNAs (10). We previously investigated miRNAs modulated by hypoxia in VSMCs and found that miR-1260b is one of the most upregulated miRNAs under hypoxia (11). miR-1260b was reported to be upregulated and promoted cell proliferation and invasion in non-small cell lung cancer cells, renal cancer cells, and HCC tissues (12-14). Thus, miR-1260b is considered a putative target for the treatment or diagnosis of such cancers. In this study, we attempted to explore the role of hypoxia-induced miR-1260b in VSMC functions.

Growth differentiation factor 11 (GDF11), a TGF- $\beta$ superfamily member, is known to regulate development and differentiation (15-17). GDF11 controls anterior-posterior patterning of the axial skeleton in mouse embryos (18). Activin type II receptors, ActRIIA and ActRIIB, mediate GDF11 signaling in axial vertebral patterning. GDF11 binds to both receptors and induces the phosphorylation of Smad2/3 (19, 20). GDF11 is also known to suppress neural stem cell proliferation by modulating the expression of multiple genes implicated in the process (17). However, the function of GDF11 in VSMCs has not been illustrated in detail.

ISSN: 1976-670X (electronic edition)

Copyright (C) 2020 by the The Korean Society for Biochemistry and Molecular Biology

(c) This is an open-access article distributed under the terms of the Creative Commons Attribution Non-Commercial License (http://creativecommons.org/licenses/by-nc/4.0) which permits unrestricted non-commercial use, distribution, and reproduction in any medium, provided the original work is properly cited. 
As enhanced proliferation of VSMCs under hypoxia can lead to vascular disorders, understanding the functions of hypoxia-induced miRNAs in the modulation of VSMC proliferation is important. In this study, we demonstrate that hypoxia-induced miR-1260b expression promotes cell proliferation. We also report that GDF11 is targeted by miR-1260b, which results in enhanced VSMC proliferation. Furthermore, we show that Smad signaling is inhibited by the modulation of the miR-1260b-GDF11 axis under hypoxia. Taken together, these results suggest that the upregulation of miR-1260b by hypoxia leads to the repression of GDF11 expression, which, in turn, results in the inhibition of Smad signaling and promotion of VSMC proliferation.

\section{RESULTS}

\section{miR-1260b is upregulated under hypoxia and promotes VSMC proliferation}

Our previous study has shown that miR-1260b is one of the most upregulated hypoxamirs in VSMCs (11). We confirmed that the expression of mature miR-1260b was significantly increased in pulmonary artery smooth muscle cells (PASMCs) after exposure to hypoxia for $24 \mathrm{~h}$ (Fig. 1A). As it is well known that hypoxia stimulates VSMC proliferation (6), we determined whether hypoxia-induced miR-1260b expression has effects on VSMC proliferation. PASMCs transfected with miR-1260b or control mimic for $24 \mathrm{~h}$ under normoxia conditions were immnuostained with anti-Ki-67 antibody to measure the number of proliferating cells. Hoechst dye was used to stain DNA in cells. Ki-67 positive proliferating cells were approximately $8 \%$ of total cells in the control mimic-transfected cells and increased to approximately $16.2 \%$ in the miR-1260b mimic-transfected cells. Quantitative

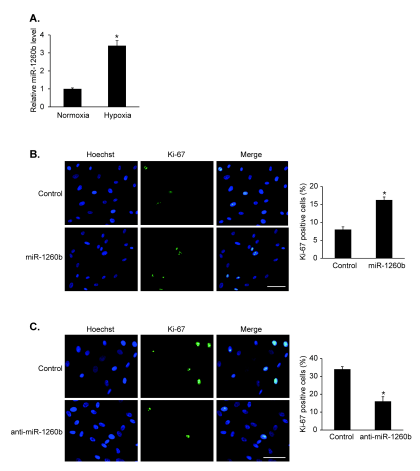

Fig. 1. Hypoxia-induced miR-1260b promotes proliferation of VSMCs. (A) Levels of miR-1260b relative to U6 snRNA measured by qRT-PCR in PASMCs $24 \mathrm{~h}$ after exposure to normoxia or hypoxia. Data represent the means \pm S.E. of triplicates. ${ }^{*} \mathrm{P}<0.05$. (B, C) Representative microphotographs of Ki-67 immunostaining. PASMCs transfected with control mimic, miR-1260b (B) or anti-miR-1260b (C) were subjected to immunofluorescence staining with anti-Ki-67 antibody. Approximately 200 cells from at least 10 independent fields were counted for each condition, and Ki-67 positive cells were presented as a percentage of the total population. Scale bar represents $50 \mu \mathrm{m} .{ }^{*} \mathrm{P}<0.05$. analysis of Ki-67 immunostaining demonstrates that miR-1260b increases the number of proliferating cells approximately 2-fold compared with the control (Fig. 1B), which suggests that miR-1260b promotes the proliferation of VSMCs. In contrast, when miR-1260b is downregulated using a hsa-miR-1260b inhibitor (anti-miR-1260b) under normoxia conditions, the percentage of proliferating cells was decreased to approximately $47 \%$ of the control mimic-transfected cells. These findings suggest that the downregulation of miR-1260b inhibits VSMC proliferation (Fig. 1C). Therefore, a hypoxia-induced increase of miR-1260b expression might stimulate VSMC proliferation.

\section{GDF11 is downregulated by hypoxia}

To further elucidate the molecular function of miR-1260b in VSMCs, we first checked whether known target mRNAs of miR-1260b, such as G-protein signaling 22, PTPRT, sFRP1, and Smad4, are downregulated by hypoxia from our NGS-based RNA sequencing data (11). None of genes investigated showed significant changes in hypoxic conditions, suggesting that hypoxia-induced miR-1260b promotes VSMC proliferation through regulation of novel targets. Thus, we searched predicted target mRNA candidates that have a conserved miRNA recognition element (MRE) sequence within their 3'UTR using a target prediction algorithm, TargetScan. We then investigated whether the expressions of the predicted target genes, such as chromosome transmission fidelity factor 8 (CHTF8), DiGeorge syndrome critical region gene 2 (DGCR2), GDF11, MAF BZIP transcription factor G (MAFG), nuclear factor I C (NFIC), RNA polymerase II subunit $\mathrm{F}$ (POLR2F), transmembrane protein 104 (TMEM104), and ubiquitin conjugating enzyme $\mathrm{E} 2 \mathrm{H}(\mathrm{UBE} 2 \mathrm{H})$, are downregulated by miR-1260b via qRT-PCR analysis. Among these 8 genes, DGCR2, GDF11 and POLR2F mRNA levels were reduced by approximately $70 \%, 60 \%$ and $37 \%$, respectively, following the overexpression of miR-1260b, which suggests that miR-1260b might regulate the expression of DGCR2, GDF11 and POLR2F (Fig. 2A). As the expression of miR-1260b is increased under hypoxia, if target genes are posttranscriptionally repressed by miR-1260b, decreased target gene expression levels would be expected under hypoxia. Thus, we examined the mRNA levels of the predicted target genes in PASMCs after 24 hours exposure to hypoxia via qRT-PCR. Under hypoxia, the transcript level of GDF11 was downregulated by approximately $58 \%$; however, no other transcript levels were significantly changed (Fig. 2B). Similar to the qRT-PCR results, GDF11 protein levels were decreased by approximately $57 \%$ in the hypoxia-exposed cells for $24 \mathrm{~h}$ (Fig. 2C). These results suggest the possibility that GDF11 might be posttranscriptionally regulated by miR- $1260 \mathrm{~b}$ under hypoxia. The overexpression of miR-1260b by the exogenous miR-1260b mimic was confirmed by qRT-PCR (Fig. 2D).

\section{GDF11 is a direct target of miR-1260b}

We examined whether endogenous GDF11 protein levels are regulated by miR-1260b. Immunoblot analyses indicated that the exogenic miR-1260b mimic significantly reduced the endogenous 


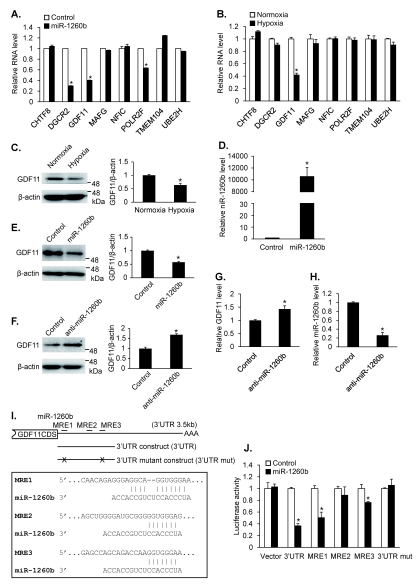

Fig. 2. Identification of target mRNAs for miR-1260b. (A) Endogenous levels of predicted target candidate mRNAs relative to $18 \mathrm{~S}$ rRNA were quantified by qRT-PCR analyses in PASMCs transfected with control or miR-1260b mimic. Data represent the means \pm S.E. of triplicates. $* P<0.05$. (B) Levels of predicted target candidate mRNA relative to $18 \mathrm{~S}$ rRNA measured by qRT-PCR $24 \mathrm{~h}$ after exposure to normoxia or hypoxia. Data represent the means + S.E. of triplicates. ${ }^{*} \mathrm{P}<0.05$. (C) Total cell lysates from PASMCs exposed to normoxia or hypoxia for $24 \mathrm{~h}$ were subjected to immunoblot analysis with anti-GDF11 or anti- $\beta$-actin antibodies. Relative amounts of GDF11 proteins normalized to $\beta$-actin were quantitated by densitometry. ${ }^{*} \mathrm{P}<0.05$. (D) Mature miR-1260b levels relative to U6 snRNA measured by qRT-PCR $24 \mathrm{~h}$ after transfection with control or miR-1260b mimic. Data represent the means \pm S.E. of triplicates. ${ }^{*} P<0.05$. (E, F) Immunoblot analysis of GDF11 and $\beta$-actin using cell lysates of PASMCs transfected with control, miR-1260b mimic (E) or anti-miR-1260b (F). Protein bands were quantitated by densitometry, and relative amounts of GDF11 proteins normalized to $\beta$-actin were presented. $* P<0.05$. (G) Levels of endogenous GDF11 mRNA relative to $18 \mathrm{~S}$ rRNA were quantified by qRT-PCR analyses in PASMCs transfected with control or anti-miR-1260b. Data represent the means \pm S.E. of triplicates. ${ }^{*} P<0.05$. $(\mathrm{H})$ Mature miR-1260b levels relative to U6 snRNA were measured by qRT-PCR $24 \mathrm{~h}$ after transfection with control or anti-miR-1260b. Data represent the means \pm S.E. of triplicates. ${ }^{*} P<0.05$. (I) (Upper panel) Schematic diagram of predicted miR-1260b MREs in the 3'UTR of GDF11 transcripts and luciferase reporter constructs used for luciferase assays. CDS and AAA represent the protein coding sequence and poly (A) tail, respectively. Mutations introduced in the MRE to disrupt a base paring with the miR-1260b sequence are indicated as X. (Bottom panel) Sequences of the predicted miR-1260b MREs in the 3'UTR of GDF11 transcripts. (J) Luciferase activities of constructs with the 3'UTR of GDF11, mutant $3^{\prime}$ UTR and MRE sequences were examined in Cos7 cells by transfecting control or miR-1260b mimic. A luciferase vector without the 3' UTR sequence (Vector) was used as a negative control. Data represent the means \pm S.E. of triplicates. $* P<0.05$.

GDF11 protein level by approximately $43 \%$ (Fig. 2E). In contrast, miR-1260b inhibition by an anti-miR-1260b elevated the basal level of GDF11 protein by approximately 1.7 -fold (Fig. 2F). The expression level of GDF11 transcripts was also elevated by approximately $43 \%$ in PASMCs transfected with an anti-miR-1260b (Fig. 2G), indicating that endogenous miR-1260b represses GDF11 expression. The inhibition of miR-1260b by anti-miR-1260b was confirmed via qRT-PCR (Fig. 2H).
We next determined whether GDF11 is targeted by a direct binding of miR-1260b via luciferase assay. Three potential miRNA recognition elements (MREs) in the GDF11 3'UTR, which are partially complementary to the miR-1260b, were found based on the prediction by TargetScan (Fig. 2l). Thus, we assessed luciferase activities of the construct that contained a partial 3' UTR of GDF11, including all three MREs, following the overexpression of the miR-1260b (Fig. 2J). The luciferase activity of the GDF11 3' UTR construct was reduced by approximately $63 \%$ in the presence of miR-1260b mimic, which indicates that GDF11 is a novel direct target of miR-1260b. To determine which of these predicted MREs are necessary for the repression by miR-1260b, luciferase reporter constructs that contained individual MREs and miR-1260b were transfected into cells. The transcriptional activity of a luciferase reporter construct that included the MRE1 or MRE3 sequence, but not MRE2, was significantly reduced by miR-1260b, which suggested that MRE1 and MRE3 are essential for the target recognition by miR-1260b. To further support that the identified MRE sequences are critical for recognition by miR-1260b, we made a mutant 3'UTR luciferase reporter construct (3'UTR mut), which disrupted both MRE1 and MRE3, and then measured the luciferase activity. The inhibition of the luciferase activity of miR-1260b is abrogated in the mutant $3^{\prime}$ UTR construct, which suggests that miR-1260b targets GDF11 by direct binding with MRE1 and MRE3 (Fig. 2J).

\section{GDF11 regulates VSMC phenotypes}

As miR-1260b promotes VSMC proliferation, we hypothesized that a target of miR-1260b, GDF11, might be linked to the modulation of cell proliferation. To examine whether the GDF11 expression affects VSMC proliferation, the number of proliferating cells was measured by immunostaining with an antibody against Ki-67 following transfection of GDF11 siRNA into PASMCs. Using siRNA, the levels of GDF11 mRNAs and proteins were reduced by approximately $78 \%$ and $60 \%$, respectively (Fig. 3A and 3B). Quantitative analysis of Ki-67 immunostaining demonstrates that knockdown of GDF11 by siRNA increased the percentage of Ki- 67 positive proliferating cells approximately 2.2 -fold compared with the control, which implies that the targeting of GDF11 by miR-1260b enhances the proliferation of VSMCs under hypoxia (Fig. 3C). We subsequently ascertained that GDF11 inhibits the proliferation of VSMCs. PASMCs were stimulated with $10 \mathrm{ng}$ of GDF11 for $24 \mathrm{~h}$ and subjected to immunostaining with an antibody against Ki-67. The number of Ki-67 positive proliferating cells was reduced by approximately 35\% following GDF11 stimulation (Fig. 3D).

As GDF11 is a secreted protein, we confirmed that secretion of GDF11 is affected by the intracellular miR-1260b levels via measuring the secreted GDF11 levels. Medium of PASMCs transfected with control miRNA or miR-1260b was used for trichloroacetic acid (TCA)-induced protein precipitation and pellets were subjected to western blotting using a GDF11 antibody. The level of GDF11 in the medium of miR-1260b-transfected cells was reduced by approximately $40 \%$ compared to that of the control 


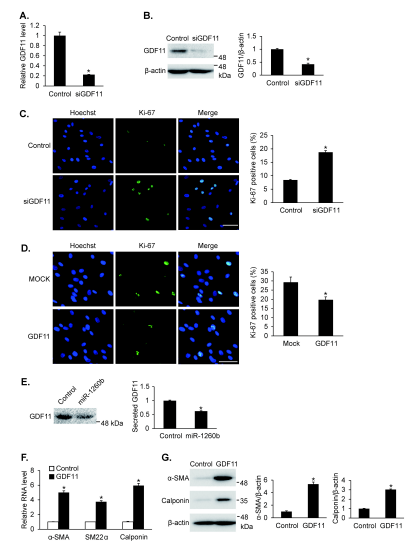

Fig. 3. GDF11 modulates VSMC proliferation. (A) Levels of endogenous GDF11 mRNA relative to $18 \mathrm{~S}$ rRNA measured by qRT-PCR analyses in PASMCs transfected with control or siGDF11. Data represent the means \pm S.E. of triplicates. ${ }^{*} P<0.05$. (B) Total cell lysates from PASMCs transfected with control or siGDF11 were subjected to immunoblot analysis with anti-GDF11 or anti- $\beta$-actin antibodies. Relative amounts of the GDF11 proteins normalized to $\beta$-actin were quantitated by densitometry. ${ }^{*} P<0.05$. (C, D) Representative microphotographs of Ki-67 immunostaining. PASMCs transfected with control or siGDF11 (C) or treated with 10 ng GDF11 (D) were subjected to immunofluorescence staining with anti-Ki-67 antibody. Approximately 200 cells from at least 10 independent fields were counted for each condition, and Ki-67 positive cells were presented as a percentage of the total population. Scale bar represents $50 \mu \mathrm{m}$. ${ }^{* P}<0.05$. (E) Proteins in the media of PASMCs transfected with control miRNA or miR-1260b for $48 \mathrm{~h}$ were precipitated by TCA-induced protein precipitation approach and subjected to western blotting using a GDF11 antibody. Bands of secreted GDF11 protein were quantitated by densitometry. $* P<0.05$. (F) Levels of smooth muscle genes relative to 18S rRNA measured by qRT-PCR analyses in PASMCs treated with 10 ng GDF11. Data represent the means \pm S.E. of triplicates. ${ }^{*} P<0.05$. (G) Protein levels of $\alpha$-SMA and calponin were measured by immunoblot analysis. Protein bands were quantitated by densitometry. ${ }^{*} \mathrm{P}<0.05$.

miRNA-transfected cells, suggesting that exogenous miR-1260b suppresses GDF11 expression and less GDF11 is secreted (Fig. 3E). These results further support that GDF11 is a key regulator of miR-1260b-mediated VSMC proliferation.

Given that proliferation is a critical feature between synthetic and contractile phenotypes of VSMCs, we investigated whether GDF11 also modulates the expression of SMC contractile-associated genes and leads to phenotypic changes of VSMCs. PASMCs were stimulated with GDF11 for $24 \mathrm{~h}$, and the mRNA levels of smooth muscle genes, such as $\alpha$-SMA, SM22 $\alpha$ and calponin, were measured via qRT-PCR. The expressions of all three smooth muscle cell-specific contractile genes were significantly increased approximately 3.7 to 6-fold following GDF11 stimulation, which suggests that GDF11 promotes the contractile phenotype (Fig. 3F). The protein levels of $\alpha$-SMA and calponin were increased approximately 5.4-fold and 3-fold, respectively, after in response to GDF11 stimulation (Fig. 3G). Considering that GDF11 inhibits the proliferation of VSMCs and enhances the expression of SMC contractile-associated genes, GDF11 induces the contractile state.

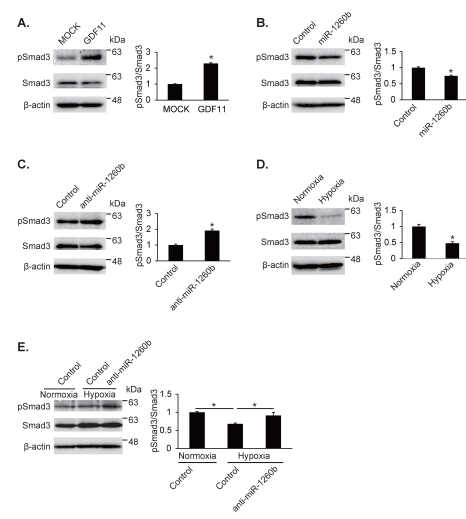

Fig. 4. Modulation of GDF11 by miR-1260b regulates Smad signaling. (A) Total cell lysates from PASMCs exposed to GDF11 for $1 \mathrm{~h}$ were subjected to immunoblot analysis with anti-phospho-Smad3, anti-Smad3 or anti- $\beta$-actin antibodies. Relative amounts of phosphorylated Smad3 normalized to total Smad3 were quantitated by densitometry. ${ }^{* P}<$ 0.05. (B, C) Total cell lysates from PASMCs transfected with control, miR-1260b mimic (B), or anti-miR-1260b (C) were subjected to immunoblot analysis with antibodies against pSmad3, Smad3 or $\beta$-actin. By densitometry, relative amounts of phosphorylated Smad3 protein normalized to total Smad3 were quantitated. ${ }^{*} P<0.05$. (D) Total cell lysates from PASMCs exposed to normoxia or hypoxia for $24 \mathrm{~h}$ were subjected to immunoblot analysis with anti-phospho-Smad3, Smad3 or anti- $\beta$-actin antibodies. By densitometry, relative amounts of phosphorylated Smad3 normalized to total Smad3 were quantitated. ${ }^{*} \mathrm{P}<0.05$. (E) PASMCs transfected with control or anti-miR-1260b were exposed to normoxia or hypoxia for $24 \mathrm{~h}$. Total cell lysates were subjected to immunoblot analysis with antibodies against pSmad3, Smad3 or $\beta$-actin. By densitometry, relative amounts of phosphorylated Smad3 normalized to total Smad3 were quantitated. ${ }^{*} \mathrm{P}<0.05$.

Therefore, miR-1260b-mediated downregulation of GDF11 under hypoxia might induce the synthetic state by modulating specific aspects of the VSMC phenotype.

\section{miR-1260b-GDF11 axis regulates Smad signaling}

As GDF11 is known to induce the phosphorylation of $S m a d 2 / 3$ in various cell types (21-24), we investigated whether the miR-1260b-GDF11 axis modulates Smad signaling in VSMCs. First, we examined the changes in Smad3 phosphoraylation following GDF11 stimulation. Immunoblot analysis using an antibody against phosphorylated Smad3 showed that the levels of phosphorylated Smad3 were enhanced in PASMCs treated with GDF11 for $1 \mathrm{~h}$, which demonstrates that GDF11 activates Smad signaling in VSMCs (Fig. 4A). Next, we examined the activation of Smad signaling after transfection of miR-1260b mimic or anti-miR-1260b into PASMCs by immunoblotting. The levels of phosphorylated Smad3 were reduced when miR-1260b was overexpressed (Fig. 4B), which suggests that miR-1260b inhibits Smad signaling. In contrast, the phosphorylation of Smad3 was enhanced following the transfection with anti-miR-1260b (Fig. 4C), which indicates that the downregulation of miR-1260b activates the Smad signaling pathway. These results imply that miR-1260b acts as a negative regulator of Smad signaling through 
the suppression of GDF11 expression.

As miR-1260b is upregulated and GDF11 is consequently repressed under hypoxia, we examined whether hypoxia affects the Smad signaling pathway. Immunoblot analysis showed a decrease of the phosphorylation levels of Smad3 in cells exposed to hypoxia for $24 \mathrm{~h}$ compared with the normoxia controls, which suggests that $\mathrm{Smad}$ signaling is inhibited under hypoxia conditions (Fig. 4D). Therefore, these results imply that Smad signaling, which can prevent the synthetic phenotype, might be impaired by the modulation of the miR-1260b-GDF11 axis under hypoxia and thus the proliferative response of VSMCs is induced.

As hypoxia-induced miR-1260b inhibits Smad signaling, we examined whether modulation of miR-1260b using anti-miR-1260b affects the hypoxia-mediated Smad signaling regulation (Fig. 4E). PASMCs transfected with control or anti-miR-1260b for $48 \mathrm{~h}$ were exposed to normoxia or hypoxia for $24 \mathrm{~h}$. In control-transfected PASMCs, the phosphorylated Smad3 level was reduced by hypoxia; however, the phosphorylated Smad3 level was recovered in anti-miR-1260b-transfected cells even under hypoxia conditions. These results suggest that modulation of miR-1260b might control Smad signaling of VSMCs under hypoxia conditions.

\section{DISCUSSION}

We previously observed that miR-1260b expression is upregulated in VSMCs exposed to hypoxia (11). However, the mechanism that underlies the regulation of hypoxic responses via miR-1260b has not been investigated. In this study, we reported miR-1260b as a novel hypoxamir to regulate VSMC proliferation and identified GDF11 as a miR-1260b target. We provided evidence that the downregulation of GDF 11 by miR-1260b is crucial for promoting proliferation under hypoxia. Furthermore, we demonstrated that Smad signaling via GDF11 is modulated by miR-1260b under hypoxia, so that VSMC proliferation is promoted.

GDF 11 , as a TGF- $\beta$ family member, is widely expressed and plays various roles, including regulating axial skeletal patterning during development (18). Recently, evidence regarding the association between GDF11 function and vascular disease has emerged. For example, the levels of plasma GDF11 were decreased in chronic obstructive pulmonary disease (COPD), in which the progression of COPD was promoted by activating the AKT signaling pathway (25). In addition, the circulating GDF11 concentration declined with age; thus GDF11 has been considered as a "rejuvenating" factor that improves cardiovascular disease, osteoporosis, and other diseases of old age, although there are conflicting reports regarding the function of GDF11 during aging $(26,27)$. However, the exact function of GDF11 associated with VSMC proliferation, which can lead to vascular disease, has not previously been elucidated. In this study, we observed that the repression of GDF11 expression inhibits Smad signaling and promotes VSMC proliferation. Our findings provide evidence for an anti-proliferative function of GDF11 and suggest that GDF11Smad3-dependent signaling is an important molecular mechanism in the regulation of VSMC proliferation. Moreover, Smad signaling upon TGF- $\beta$ family ligands is known to be critical to regulate VSMC phenotypes through the inhibition of cell proliferation (4).

We previously demonstrated that BMP4-Smad signaling induces miR-21 biogenesis, which increases contractile gene expression and inhibits cell migration by targeting PDCD4 and DOCK family proteins, respectively, thus promoting the contractile phenotype of VSMCs $(28,29)$. In addition, BMP4-Smad signaling downregulates miR-302 that targets the BMP receptor type II (BMPRII) as an autoregulatory mechanism, which inhibits cell migration and proliferation and accelerates the contractile phenotype of VSMCs (30). In this study, we identified an additional miRNA, miR-1260b, which regulates VSMC functions, further supporting the fact that miRNAs provide a delicate regulatory mechanism in the complex vascular system. As Smad proteins are the main signal transducers for the TGF- $\beta$ signaling pathway that inhibits proliferation of VSMCs, suppression of GDF11 as a member of the TGF- $\beta$ superfamily by miR- $1260 \mathrm{~b}$ impairs the TGF- $\beta$ signaling pathway, and consequently VSMC proliferation is accelerated.

Several studies suggest that miR-1260b is implicated in the proliferation, migration or invasion of cancer cells (12-14); however the role of miR-1260b in VSMCs is unclear, and the function associated with hypoxia conditions has not previously been elucidated. We first report miR-1260b as a hypoxamir. miR-1260b expression is induced by hypoxia and has a proproliferative function in VSMCs. Hypoxia-induced miR-1260b might contribute to promoting VSMC proliferation by targeting GDF11 to inhibit Smad signaling that restrains the proliferative synthetic phenotype. As hypoxia-induced pulmonary artery hypertension and many other vascular diseases are associated with increased VSMC proliferation, miR-1260b is likely to be a crucial mediator to develop vasculopathies. Our understanding of the regulatory functions of miR-1260b in the proliferation of VSMCs under hypoxia provides new insight into the mechanisms of vascular proliferative disorders.

\section{CONCLUSION}

Several lines of evidence have converged to link GDF11 with vascular diseases. Our findings raise the possibility that the hypoxia-induced proliferative response of VSMCs might be mediated by modulation of the miRNA-GDF11 axis. miR-1260b appears to be of biological importance because it was upregulated by hypoxia and promoted VSMC proliferation. Our finding of GDF11 as a target of miR-1260b provides relevant insight into the mechanisms of hypoxia-induced vascular proliferative pathologies and potential future interventions.

\section{MATERIALS AND METHODS}

Detailed information is provided in the Supplementary Information. 


\section{ACKNOWLEDGEMENTS}

This work was supported by the Incheon National University Research Grant in 2016.

\section{CONFLICTS OF INTEREST}

The authors have no conflicting interests.

\section{REFERENCES}

1. Owens GK, Kumar MS and Wamhoff BR (2004) Molecular regulation of vascular smooth muscle cell differentiation in development and disease. Physiol Rev 84, 767-801

2. Owens GK (1995) Regulation of differentiation of vascular smooth muscle cells. Physiol Rev 75, 487-517

3. Lagna G, Ku MM, Nguyen PH, Neuman NA, Davis BN and Hata A (2007) Control of phenotypic plasticity of smooth muscle cells by bone morphogenetic protein signaling through the myocardin-related transcription factors. J Biol Chem 282, 37244-37255

4. ten Dijke P and Arthur HM (2007) Extracellular control of TGFbeta signalling in vascular development and disease. Nature reviews. Mol Cell Biol 8, 857-869

5. Semenza GL (2007) Life with oxygen. Science 318, 62-64

6. Stenmark KR, Fagan KA and Frid MG (2006) Hypoxiainduced pulmonary vascular remodeling: cellular and molecular mechanisms. Circ Res 99, 675-691

7. Semenza GL (2012) Hypoxia-inducible factors in physiology and medicine. Cell 148, 399-408

8. Wang GL, Jiang BH, Rue EA and Semenza GL (1995) Hypoxia-inducible factor 1 is a basic-helix-loop-helix-PAS heterodimer regulated by cellular $\mathrm{O} 2$ tension. Proc Natl Acad Sci U S A 92, 5510-5514

9. Ivan M, Harris AL, Martelli F and Kulshreshtha R (2008) Hypoxia response and microRNAs: no longer two separate worlds. J Cell Mol Med 12, 1426-1431

10. Kang H and Hata A (2012) MicroRNA regulation of smooth muscle gene expression and phenotype. Curr Opi Hemato 19, 224-231

11. Lee J, Heo J and Kang $\mathrm{H}$ (2018) miR-92b-3p-TSC1 axis is critical for mTOR signaling-mediated vascular smooth muscle cell proliferation induced by hypoxia. Cell Death Differ 26, 1782-1795

12. Hirata H, Ueno K, Nakajima K et al (2013) Genistein downregulates onco-miR-1260b and inhibits Wnt-signalling in renal cancer cells. Br J Can 108, 2070-2078

13. Li X, Song H, Liu Z and Bi Y (2018) miR-1260b promotes cell migration and invasion of hepatocellular carcinoma by targeting the regulator of G-protein signaling 22. Biotech Lett $40,57-62$

14. $\mathrm{Xu} \mathrm{L}, \mathrm{Xu} X$, Huang $\mathrm{H}$ et al (2018) MiR-1260b promotes the migration and invasion in non-small cell lung cancer via targeting PTPRK. Pathol Res Prac 214, 776-783

15. Kim J, Wu HH, Lander AD, Lyons KM, Matzuk MM and Calof
AL (2005) GDF11 controls the timing of progenitor cell competence in developing retina. Science 308, 1927-1930

16. Nakashima M, Mizunuma K, Murakami T and Akamine A (2002) Induction of dental pulp stem cell differentiation into odontoblasts by electroporation-mediated gene delivery of growth/differentiation factor 11 (Gdf11). Gene Ther 9, 814818

17. Wu HH, Ivkovic S, Murray RC et al (2003) Autoregulation of neurogenesis by GDF11. Neuron 37, 197-207

18. McPherron AC, Lawler AM and Lee SJ (1999) Regulation of anterior/posterior patterning of the axial skeleton by growth/differentiation factor 11. Nat Genetics 22, 260-264

19. Andersson O, Reissmann E and Ibanez CF (2006) Growth differentiation factor 11 signals through the transforming growth factor-beta receptor ALK5 to regionalize the anteriorposterior axis. EMBO Rep 7, 831-837

20. Oh SP, Yeo CY, Lee Y, Schrewe H, Whitman M and Li E (2002) Activin type IIA and IIB receptors mediate Gdf11 signaling in axial vertebral patterning. Gene Dev 16, 2749-2754

21. Gaunt SJ, George M and Paul YL (2013) Direct activation of a mouse Hoxd11 axial expression enhancer by Gdf11/Smad signalling. Devel Biol 383, 52-60

22. Hannan NR, Jamshidi P, Pera MF and Wolvetang EJ (2009) BMP-11 and myostatin support undifferentiated growth of human embryonic stem cells in feeder-free cultures. Clon Stem Cells 11, 427-435

23. Suragani RN, Cadena SM, Cawley SM et al (2014) Transforming growth factor-beta superfamily ligand trap ACE-536 corrects anemia by promoting late-stage erythropoiesis. Nat Med 20, 408-414

24. Williams G, Zentar MP, Gajendra S, Sonego M, Doherty P and Lalli G (2013) Transcriptional basis for the inhibition of neural stem cell proliferation and migration by the TGFbeta-family member GDF11. PLoS One 8, e78478

25. Tang F, Ling C and Liu J (2018) Reduced expression of growth differentiation factor 11 promoted the progression of chronic obstructive pulmonary disease by activating the AKT signaling pathway. Biomed Pharmacother 103, 691-698

26. Fan X, Gaur U, Sun L, Yang D and Yang M (2017) The Growth Differentiation Factor 11 (GDF11) and Myostatin (MSTN) in tissue specific aging. Mech Age Devel 164, 108-112

27. Poggioli T, Vujic A, Yang P et al (2016) Circulating Growth Differentiation Factor 11/8 Levels Decline With Age. Cir Res 118, 29-37

28. Davis BN, Hilyard AC, Lagna G and Hata A (2008) SMAD proteins control DROSHA-mediated microRNA maturation. Nature 454, 56-61

29. Kang H, Davis-Dusenbery BN, Nguyen PH et al (2012) Bone morphogenetic protein 4 promotes vascular smooth muscle contractility by activating microRNA-21 (miR-21), which down-regulates expression of family of dedicator of cytokinesis (DOCK) proteins. J Biol Chem 287, 3976-3986

30. Kang H, Louie J, Weisman A et al (2012) Inhibition of microRNA-302 (miR-302) by bone morphogenetic protein 4 (BMP4) facilitates the BMP signaling pathway. J Biol Chem 287, 38656-38664 